\section{EMOTIONAL INTELLIGENCE: USE IN MEDICAL EDUCATION AND PRACTICE}

Cognitive intelligence (IQ) evaluates cognitive capacities as a general fund of knowledge, analytical and logical thinking, capacity to remember and recall, and the capacity to compute accurately (1). IQ characterizes "book smarts" individuals, but has a weak link in predicting success. Beside strong intellectual abilities, medical students and physicians should therefore acquire and control emotional intelligence (EI). EI depicts dimensions of intelligence significant in successfully dealing with daily environmental pressure: self-awareness, control of emotions, relationships, and enlightened and progressive communication. Thus, EI helps to predict success and characterizes "street smarts".

EI does not drive out IQ; human poly-intelligence does exist. EI allowed researchers to pay tribute to the complexity, richness, sensitivity, and depth of the multidimensionality of the human being. This polyintelligence enables physicians to assume responsibilities and control complexity in an optimal way.

Five main components concerning the medical field describe EI (1). Intrapersonal components include emotional self-awareness, assertiveness, self-regard, self-actualization, and independence. Similarly, interpersonal components include empathy, social responsibility and interpersonal relationships. In addition, adaptability is highlighted by problem solving, reality testing and flexibility; and stress management can be described by stress tolerance and impulse control; and general mood is shown through happiness and optimism. These components are essential for medical leadership and involve concrete results in medical education, professional relationships and development, and patients' care.

Emotions should be coupled to teaching since emotions strongly influence behaviors. Instead of attending lectures as a passive audience member, the student discovers knowledge through a deeper dimension as he recognizes the value of the lecturer's messages. Didactic instruction which activates emotions and feelings inclines students to learn and improves their performance. Integrating EI training into graduate medical education may accordingly improve young physicians' interpersonal and communication skills while creating a more caring environment for patients (2).

In the care team, the physician using EI facilitates a healthy dialogue by attentive listening and, with adequate emotional serenity, harnesses and optimizes interpersonal relationships (3). An adequate control of emotions makes possible to be functional in every situation and to make rational decisions, thus acting as a mentor for residents and students for becoming more effective on the ward and in communication. The EI physician makes his colleagues aware on what can be created collectively, by direct encouragement, realistic expectations, and tangible opportunities to learn new skills, highlighting the purpose of personal liabilities, initiative and innovation (4). The physician acts so to stimulate creativity, avoiding criticism and feeling less threatened by changes resulting from creative ideas. The EI physician answers in a constructive way to changes and takes advantage of these new opportunities (5).

EI offers a teaching framework that allows learning from collaborative relationships in order to increase professional development and leadership. Emotional learning and maturation are paramount to both. EI is a quality that improves and optimizes by personal reflection, practice and supervision. With colleagues, the EI physician consolidates therefore a supportive work climate, encouraging high-efficiency, in which other health professionals feel energized to perform get better in a mutual confidence ambiance (4).

Moreover relationships between colleagues, EI training could lead to better work/life balance and doctor-patient relations. EI physician builds resilience and copes with stressful periods, as well as experiences better physical and psychological health (6). Therefore, physicians care for themselves through self-awareness and reflection, which appears to be one of the most important things to provide good care to others. Being more sensitive to their own and their patients' feelings and emotions, EI physicians use supportive behavior and provide psychological benefits (5).

EI skills are mainly developed trough life's experience. Students and physicians should use introspection. Introspection is a goal for professional and private life success. One should analyses and learns from its own gestures, speech and behaviors. Leadership literature (7) is also useful for EI improvement, as both entities are highly related. 
Medical associations as the Canadian Medical Association (CMA) may offer non-clinical professional development to medical students, fellows and physicians. For instance, 8 out of the 17 Canadian medical schools are currently piloting the CMA Student Leadership Curriculum (7). This program teaches selfawareness and performance improvement, healthy personal and professional growth, effective communication, team building skill development, and conflict resolution.

Leaders challenge remains in becoming familiar with a powerful combination made of knowledge and relation management. Emotional intelligence can be learned, developed, and reinforced with life experience. EI is accessible if we decide to consider it to be a resource and not a sentimental weakness, but only if we support its emergence in our daily lifestyle.

Sincerely,

Jimmy Bejjani

University of Sherbrooke

Jimmy.Bejjani@USherbrooke.ca

\section{REFERENCES}

1. Stein S., Book H. The EQ Edge: Emotionnal Intelligence and Your Success. Wiley Publishing. 2002

2. Cassels C., Emotional Intelligence Training Could Lead to Better Doctor-Patient Relations. JAMA, 2008;12:1200-1202.

3. Freshman B., Rubino L. Emotional intelligence skills for maintaining social networks in health organizations. Hospital Topics: Research and Perspectives on Healthcare. 2004; 82(3);2-9.

4. Yoder D.M., Organizational climate and emotional intelligence: an appreciative inquiery into a "leaderful" community college. Community College Journal of Research and Practice. 2005; 29;45-62.

5. $\quad$ Rego A., Sousa F., Pina a Cunha M., et al., Leader self-reported emotional intelligence and perceived employee creativity and perceived employee creativity. Creativity and Innovation Management, 2007;16 (3);250-264.

6. Ciarrochi J., Scott G., The link between emotional competence and well-being: a longitudinal study. British Journal of Guidance \& Counselling, 2006;34 (2);231-243.

7. CMA Office for Leadership and Professional Development. URL: www.cma.ca/leadership

Jimmy Bejjani (MD 2010) is studying medicine at the University of Sherbrooke, QC. He has an interest, and has been actively involved, in surgical-related studies since 2005 